\title{
Algunas peculiaridades de la ciencia bibliotecológica
}

\author{
Miguel Ángel Rendón Rojas \\ Investigador del Centro Universitario de \\ Investigaciones Bibliotecológicas de la \\ UNAM. Torre II de Humanidades, piso 12 , \\ Ciudad Universitaria, México D.F. tel: 623 \\ 0343 \\ E-mail:marr@servidor.unam.mx
}

\begin{abstract}
RESUMEN
$\boldsymbol{E}_{1}$ autor afirma que existen elementos objetivos (la lógica, la sintaxis y la tecnología) y subjetivos (el Ser humano) dentro de la ciencia bibliotecológica, por lo que es posible un enfoque positivista y uno hermenéutico. Se toma la segunda posibilidad y se expresa que la bibliotecología cae dentro de las ciencias sociales, se analizan las consecuencias de esta última afirmación, subrayándose el papel que la axiología juega dentro de ese cuerpo de conocimiento. Se indica que el objeto de estudio de la bibliotecología es la relación entre el mundo de la información al que conduce un documento, el sujeto que desea ingresar al mundo de la información mediante un documento para satisfacer una necesidad de información y la institución informativa que da las condiciones necesarias para que el sujeto ingrese al mundo de la información. Se proporciona el análisis de esos elementos y su justificación filosófica. Por último, se distinguen diversas disciplinas de acuerdo con la parte del mundo de la información que se estudie, los medios y los fines que se emplean para llegar a él.
\end{abstract}

\section{ABSTRACT}

$\boldsymbol{T}$ he author states that exists objective elements (logics, syntaxis and technology) and subjetive elements (the human being) within library science, therefore, it is being possible a positivistic viewpoint and a hermeneutic viewpoint. The second possibility is chosen and it is expressed that library science falls within social sciences. The consecuences of this last statement are analyzed, outlining the role axiology plays in this body of knowledge. It is pointed out that the object of study of library science is the relation among the information world to which a document leads, the subject who wish to get into this information world through a document for satisfying an information need and the informative institution which gives the necessary conditions so that the subject may get into the world of information. This elements of analysis are provided as well as its historical racionality. Lastly, diverse disciplines are view as separate entities according to the information world part that is being studied, as well as the media and goals employed for reaching it.

$\boldsymbol{A}$ nte la pregunta sobre la cientificidad de una disciplina, se debe, entre otras cosas, determinar a qué campo del conocimiento humano pertenece ese cuerpo teórico analizado; es decir, utilizando el lenguaje de Dilthey, se debe establecer si es una ciencia natural o una ciencia del espíritu ${ }^{1}$. Así pues, al cuestionarse sobre el status epistemológico de la bibliotecología nos enfrentamos a ese problema ¿la bibliotecología pertenece a las ciencias naturales o a las ciencias sociales y humanísticas?

Es evidente que los fenómenos y objetos analizados en esta disciplina (recordemos los mencionados en la introducción de esta mesa: documento, información, sujeto, institución, entre otros) no son na- turales, sino que son creados, son culturales en el sentido estricto etimológico de la palabra, es decir, son cultivados, y por lo tanto están íntimamente relacionados con el hombre y con la sociedad. Pero por otro lado, esos objetos y fenómenos también están unidos al lenguaje y por medio de él, al pensamiento, a través del cual se conectan con la lógica, que es una ciencia formal apodíctica y universal; el mismo lenguaje, mediante su análisis sintáctico, da la oportunidad de realizar estudios impersonales (no-subjetivos), además de que la aplicación de la tecnología, con base en la lógica y en la sintaxis, permite establecer relaciones totalmente objetivas. Esos tres elementos (lógica, sintaxis y tecnología) y quizá haya más, dan pie a suponer la existencia de elementos independientes de la sociedad y del ser humano en el área de la bibliotecología.

De esta dualidad surge un dilema: abandonar el origen humano y hacer a la ciencia "objetiva", "natural", "racional" o reconocer ese elemento humano y entonces, se vuelven a abrir dos posibilidades: se declara la no-cientificidad de la disciplina por su subjetividad o se adopta un enfoque que acepte la cientificidad de las ciencias del espíritu, lo que implica cambiar el concepto de ciencia y abrir la puerta de "esa cosa llamada ciencia" a la teleología, a la racionalidad dialógica, a la comprensión, a la dimensión hermenéutica. 
En ocasiones, por reminiscencias positivistas se niega a aceptar el elemento humano en el cuerpo del conocimiento bibliotecológico, porque eso nos llevaría, dentro de ese modo de pensar positivista, a declarar que la disciplina no es ciencia, por lo que, el mecanismo de defensa nos empuja a tener preferencia por afirmar la objetividad pura de la teoría, lo que significa afirmar su cientificidad. En este caso, se centra la atención en los elementos no-subjetivos que enunciamos anteriormente: la lógica, la sintaxis y la tecnología.

Sin embargo, tal posición no puede negar un hecho dado: el origen, el fin y el centro de la acción bibliotecológica tienen un elemento humano-social; el medio, la forma, los instrumentos son objetivos, cosa que tampoco se puede negar, ellos cumplen una función importante y necesaria, se deben atender, estudiar, desarrollar, ellos también entran en el objeto de la bibliotecología, pero no se debe olvidar su mediatez.

De antemano diremos que nuestra posición no concuerda con esa visión positivista de la ciencia y reconocemos la posibilidad de las ciencias humanas con su especificidad, diferenciándose de las ciencias naturales por su función epistemológica. Los enunciados de las Ciencias del Espíritu también son verdaderos, entendiendo el concepto de verdad de una manera clásica aristotélica, como correspondencia ${ }^{2}$ Pero esos enunciados más que explicar y predecir (cosa que también pueden hacer a su manera) y manipular (lo que ya no hace tanto), tienden a la comprensión y descansan en una axiología, que no por ser "humana demasiado humana" es subjetiva, sino objetiva en el sentido de que se desprende de una estructura ontológica de los sujetos que la aceptan.

La base axiológica es el eje rector de la disciplina, la que nos debe orientar en el torbellino de cambios, y con base en la cual podemos valorar las tradiciones y las innovaciones, escogiendo cuáles po- demos adoptar y conservar y cuáles desechar ${ }^{3}$ Dicha base axiológica no será objeto de discusión en esta mesa, tampoco lo será la crítica a la visión positivista de la ciencia; sólo baste por el momento observar las consecuencias teóricas y prácticas de esa filosofía de la ciencia y la ausencia de axiología rectora. En el plano teórico, el racionalismo clásico no encontró respuestas convincentes a cuestiones centrales, tales como el problema de la inducción, la relación entre marco teórico y empírico, la reducción de los términos teóricos a términos empíricos, y otros; por lo que actualmente se encuentra en crisis y se enfrenta al posmodernismo, que en sus corrientes extremas regresan al irracionalismo. Asimismo, recibe la crítica de la escuela de Frankfurt que declara el afán de dominio y manipulación escondido bajo el manto de la objetividad, afirmando que esa racionalidad no es más que una racionalidad instrumental. Así pues, observamos que se desarrolla en lugar del neo o postpositivismo una serie de filosofías de la ciencia de tendencia hermenéutica, desde Heidegger y Gadamer hasta Habermas y Apel. En el plano de la práctica, la crítica al positivismo, en su acepción de Ilustración, no puede ser más arrolladora; esa ciencia objetiva no cumplió con su programa ético-político en el área social, no explicó todo lo que prometió explicar y el poder que otorgó la ciencia se convirtió en dominio elitista de los expertos.

Por otro lado, la ausencia de una axiología rectora ha ocasionado depender de coyunturas políticas y económicas, ir detrás, principalmente, de tecnologías en el área de la ingeniería de la información, sin reflexionar qué atañe a un bibliotecólogo y qué no. No es posible que la actividad bibliotecológica esté supeditada a los designios de un dirigente "humanista”, pero que el día de mañana puede ser sustituido por un "tecnócrata", el que a su vez le puede dejar el lugar a un "fascista", y de esta manera se cambie de finalidades continuamente; asimismo, no es posible que todo dependa de los "buenos bibliotecarios". Debe existir una axiología clara y fundamento de la disciplina. 4

Así pues, al aceptar que la bibliotecología es una disciplina social, ${ }^{5}$ debemos reconocer sus propiedades específicas; es necesario dejar de compararla con las ciencias naturales, en ella no podemos encontrar enunciados nomológicos categóricos y cuantificables; no vamos a encontrar relaciones del tipo

$$
V=\frac{d}{t} ; E=m c^{2} ;
$$

o leyes como la de gravitación de Newton o la ley de Coulomb; por el contrario, los enunciados generales de la bibliotecología tienen un carácter teleológico, indican tendencias y son de carácter hipotético. De esta manera encontramos proposiciones tales como "la actividad informativa-documental comienza con una necesidad de información", o "si la información obtenida equivale a la información solicitada y ésta satisface la necesidad de información, entonces se cierra el ciclo de la actividad informativodocumental con ese usuario". La historia y la sociología trabajan con proposiciones semejantes, así tenemos el siguiente enunciado: "si existe una crisis económica y no hay confianza en el poder político, entonces puede empezar una crisis social". Ese enunciado también es de carácter hipotético ya que expresa una posible tendencia, pero que no es necesario, ya que diversos factores contingentes influyen en su actualización; por ejemplo en México, hemos pasado varias crisis económicas, nunca ha habido confianza en el poder político, pero no ha estallado una crisis social. 6

Ahora bien, el hecho de que la bibliotecología sea una ciencia social no implica que proporcione un tipo de conocimiento relativo, en el sentido de subjetivo; como ya se indicó, en la disciplina existen enunciados verdaderos, capaces de de-

2 Decimos que un enunciado es verdadero si y sólo si el estado de cosas que expresa se da en la realidad.

3 Además de que el fundamento axiológico de la disciplina se desprende igualmente de su naturaleza práctica. Siguiendo a Husserl, concluimos que toda disciplina práctica descansa en un sistema de valores, con base en el cual se fundamentan las acciones propuestas por dicha disciplina.

4 Eso será tema de otra mesa en este Coloquio.

5 Posición que comparte, por ejemplo, Chubarián. Cfr. O. S. Chubarián. Bibliotecología. La Habana: Editorial Científico-Técnica, 1976. p. 9.

6 El mismo Marx, quien pretendió descubrir las verdaderas leyes que rigen el desarrollo social, reconoció la importancia de otros elementos y condiciones en el funcionamiento de esas leyes, por lo que no se podía hablar de regularidades universales y necesarias; señala que la presencia de ciertos factores semejantes en dos sociedades conducen en ocasiones a resultados diferentes. 


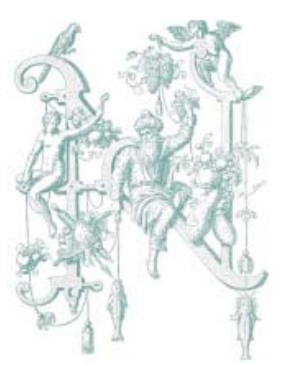

\section{De acuerdo con nuestro punto de vista, la información es una cualidad secundaria de los objetos, que es elaborada por los sujetos a partir de la estructuración de propiedades presentes en esos objetos. Como resultado de esa estructuración se crea un ente ideal, que se objetiviza y llega a formar el mundo de la información. De tal manera, el mundo de la información se concibe como ideal, pero objetivo}

mostrarse o verificarse; además de que los elementos objetivos de los que hablamos anteriormente le dan a las ciencias de la información un carácter peculiar, por ejemplo, un sistema de clasificación cumple con todos los atributos de un sistema sintáctico y semántico, es consistente y completo; entre las subclases de ese sistema se cumplen los teoremas de la teoría de conjuntos, tal vez es posible axiomatizar ese sistema por medio de la Mereología, etcétera. Es decir, existen enunciados verdaderos, independientemente de la voluntad del hombre, es por esto, que algunos han llegado a la idea de que aparte de las ciencias naturales y humanas existen las ciencias de la información, que se encuentran en la frontera de las anteriores, y que tienen características de ambas.

Dejando por sentado la naturaleza humana-social de la disciplina, adentrémonos en el problema gnoseológico central de cada teoría, a la cuestión de su objeto de estudio. Considero que para tratar el problema del objeto de estudio de la bibliotecología es necesario partir de la relación entre tres elementos esenciales: el mundo de la información, un Sujeto que desea adentrarse en ese mundo de la información y una Institución que proporciona los elementos necesarios y suficientes para que el Sujeto ingrese en el mundo de la información. Analicemos brevemente esos tres elementos.

De acuerdo con nuestro punto de vista, la información es una cualidad secundaria de los objetos, que es elaborada por los sujetos a partir de la estructuración de propiedades presentes en esos objetos. Como resultado de esa estructuración se crea un ente ideal, que se objetiviza y llega a formar el mundo de la información. De tal manera, el mundo de la información se concibe como ideal, pero objetivo. Es creado, pero no inventado, es secundario y no platónico, depende del sujeto en su creación y existencia. De esta manera, tratamos el concepto de "información" no de una manera sintáctica como en la teoría matemática de la información, ni de una manera semántica al estilo de la teoría semántica de la información de Carnap y Bar-Hillel, sino pragmáticamente, en donde el papel del sujeto es esencial. En el mundo de la información habitan, empleando la terminología de Frege, el sentido de las expresiones, o las intenciones del alma, dirían los medievales o fenomenólogos contemporáneos, los nexos que relacionan al signo con su referente, los significados de los enunciados, las formas lógicas del pensamiento, las relaciones entre esas formas del pensamiento, los postulados de significación. Ese mundo da unidad y generalidad a lo individual, da las condiciones para la comunicación y la traducibilidad. ${ }^{7}$

El Sujeto, con sus necesidades de información, es el agente principal que origina y es el fin de la actividad informativa. Para satisfacer dichas necesidades de información, exige o puede exigir ingresar a determinado fragmento del mundo de la información. Precisamente, en el Sujeto descansa la fundamentación ontológica de la objetividad de la acción informativa. Heidegger expresa que el ser del hombre se caracteriza, no por lo que es, sino porque siempre está en proceso de ser, siempre está haciendo su ser. El hombre es un ser histórico que se desarrolla a través del tiempo, incorporando en su proyecto existencial cosas y a otras personas.

Se ha preguntado el porqué la necesidad de guardar documentos, el porqué de la memoria social, la respuesta es sencilla: para Ser. Si el hombre es histórico significa que tiene un pasado y un porvenir; sin memoria no hay pasado y sin éste no se puede construir el futuro. Heidegger habla de que para estructurar un proyecto existencial se necesita una "pre-comprensión", que es el patrimonio de ideas, tradiciones, cultura, sin ella no es posible establecer un proyecto existencial. Esa pre-comprensión es dada por la actividad bibliotecológica (y por otras: la pedagógica, el habla, etcétera).

El papel que juega la memoria social explica el temor de las sociedades primitivas, que no conocían la escritura, a que se perdiera el patrimonio cultural que les daba identidad y ser; para evitar esa pérdida, utilizaban recursos como poemas, cantos, leyendas, relatos que se transmitían de generación en generación. Este hecho también nos proporciona la opor-

7 Para tener una visión más completa sobre este punto se puede consultar mi artículo "La información como ente ideal objetivizado." En Investigación bibliotecológica, Vol. 9, No. 18. pp. 17-24. 
tunidad de interpretar el fenómeno de que la escritura era considerada como algo sagrado $^{8}$ : la divinidad les dio el ser y posteriormente la escritura les permitía conservarlo. Éstas no son divagaciones abstractas; recuérdese la destrucción de documentos ordenados por Tlacaélel, la Inquisición o Stalin. Ellos sabían que si se destruía su patrimonio cultural, sería más fácil dominar a los pueblos sometidos, porque sencillamente ellos perderían su identidad. ¿Quién conoce la cosmovisión de los pueblos conquistados por los aztecas? ¿por qué se derrumbó la Unión Soviética sin cataclismos? porque no tenía pasado, su pasado era un pasado donde dominaba la explotación y tenía que ser olvidado, no había raíces y por lo tanto no tenía en qué apoyarse y con qué nutrirse.

El tercer elemento central que está en el foco de atención de la bibliotecología es la Institución, la cual abre las puertas al Sujeto para que éste ingrese en el mundo de la información. Hemos expresado la idea de que la información creada por el Sujeto se objetiviza en un ente ideal, pero también se objetiviza en un ente material: el documento. El documento es $\lambda \mathrm{o}-$ yos un en su ser-inauténtico. Por lo tanto, la Institución es un agente que no sólo resguarda las objetivaciones materiales del espíritu humano, la cultura objetivada en registros, sino que es el lugar que da las condiciones para la desobjetivación del espíritu humano. Desobjetivación, en cuanto esos documentos, al ser consultados por un sujeto, se convierten una vez más en $\lambda$ oyos en ser-auténtico, en ente ideal; pero también desobjetivación en el sentido de que esa información ya no está frente al Sujeto, sino en el Sujeto. Utilizando terminología hegeliana, no hay enajenación del espíritu humano, sino "autoconocimiento." 9

La Institución no sólo es memoria pasiva, sino capacidad activa $\delta v v \alpha \mu \iota \varsigma$, ya no es un espacio físico tridimensional, por lo que nos podemos desprender del nexo institución-edificio, para dar cabida a otro tipo de instituciones, como las bibliotecas ambulantes, móviles, e incluso electrónicas y virtuales, si es que ellas realmente pueden proporcionar las condiciones necesarias para que se realice esa desobjetivación del espíritu humano. Esa institución, siguiendo una vez más a Heidegger, es la casa del Ser, el cual sale al encuentro del hombre para que se realice la develación de la verdad, la $\alpha \lambda \eta \theta \varepsilon \iota \alpha$.

Podemos diferenciar distintas disciplinas que estudian el mundo de la información, tomando en cuenta qué parte de él estudian, cuáles datos son los que utilizan para llegar a ese mundo, qué finalidad persiguen al conectar al hombre con ese mundo y qué institución realiza ese enlace.

Así por ejemplo, las ciencias de la comunicación estudian cómo se conecta el hombre con ese mundo para dárselo a conocer a otros hombres; el periodismo se propone, a partir de hechos, producir información y crear opinión pública. Si el punto de partida son las obras de arte o las obras históricas, que también nos pueden llevar al mundo de la información, aunque no es su cometido principal, entonces tenemos la Museología, cuyo objetivo es producir un sentimiento estético o un conocimiento histórico.

Pero si se trabaja con documentos, con un lenguaje lógico y articulado, entonces, aparecen la bibliotecología, la archivología y la ciencia de la información. La bibliotecología se interesa porque los usuarios, que aparecen en el papel de lectores, tengan acceso al mundo de la información que ciertos documentos (fuentes primarias) los pueden conducir y la institución que realiza esa función es la biblioteca. Precisamente conocer las leyes que rigen esa comunicación de los lectores con la "noosfera" a través del fondo bibliotecario y las actividades que deben realizarse para que se dé esa relación es tarea de la bibliotecología.

La archivología centra su atención en ciertos documentos, distintos de los que le interesan a la bibliotecología, y conduce a otro segmento del mundo de la información, más bien de carácter histórico, donde el archivo es el encargado de realizar esa tarea.

\section{Podemos diferenciar distintas disciplinas que estudian el mundo de la información, tomando en cuenta qué parte de él estudian, cuáles datos son los que utilizan para llegar a ese mundo, qué finalidad persiguen al conectar al hombre con ese mundo y qué institución realiza ese enlace}

8 Hagamos abstracción por el momento de factores económicos y políticos que pueden explicar la prohibición de que el pueblo tuviera acceso al conocimiento como un recurso de los gobernantes para perpetuar su poder.

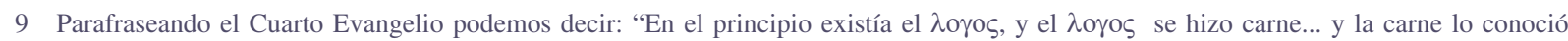
haciéndolo otra vez $\lambda$ oyos. 
La ciencia de la información centra su atención en el análisis de documentos que nos llevan a una parte del mundo de la información: la información científica, misma que puede proporcionar nueva información que no estaba presente en los documentos iniciales, descubrir regularidades, etcétera.

Considero que este enfoque permite poner en evidencia ciertos aspectos importantes que en ocasiones pasan inadverti- dos. La información puede ser muchas cosas, desde mercancía, hasta antientropía y contenidos de proposiciones, pero lo que nos interesa es una información que satisfaga las necesidades de información de un sujeto, que será una información de tipo pragmático.

La institución ya no está ligada a algo físico sino a un $\tau 0 \pi$ o en el que se ofrecen ciertas condiciones, para lo que es nece- sario realizar ciertas actividades (conservación, organización, servicio, etcétera). Dentro de esta concepción encontramos enunciados generales de tipo hermenéutico y explicativo, comprensivos y causales. La discusión puede continuar, pero sólo para que la comprensión pueda ser ampliada.

\section{BIBLIOGRAFÍA}

Bar-Hillel, Y. y R. Carnap. "Semantic Information". En Introduction to Information Science. Comp. y ed. by Tekfo Saracevic. New York \& London: Browker Company, 1979. pp. 28-23.

Bofill, J. y otros. Epistemología de la comunicación. Valencia: Fdo. Torres ed., 1976.

Chubarián, O.S. Bibliotecología General. La Habana: Editorial Científico Técnica, 1981.

Dilthey, W. Introducción a las ciencias del espíritu. Madrid: Alianza, 1980.

Hiedegger, M. El ser y el tiempo. México: FCE, 1980.

Kuhn, T. Segundos pensamientos sobre paradigmas. Madrid: Tecnos, 1978.

Rendón Rojas, Miguel Ángel. "La información como ente ideal objetivizado." En Investigación bibliotecológica. Vol. 9 No. 18. pp. 17-24. México: UNAM-CUIB, 1995.

Setién Quezada, Emilio y Salvador Gorbea Portal. "De la bibliotecología al sistema de conocimientos bibliológico informativos." En Investigación bibliotecológica. Vol. 8 No. 16 pp. 21-25. México: UNAM-CUIB, 1994.

Shannon, C.E. y W. Weaver. The Mathematical Theory of Communication. Urbana: University Press, 1962.

Vattimo, G. Introducción a Heidegger. Barcelona: Gedisa, 1986.

Wright, von G.H. Explicación y comprensión. Madrid: Alianza, 1980. 